\title{
Effect of Magnetic Field on Resistive Transition of Thin Film Thallium Based Superconductors
}

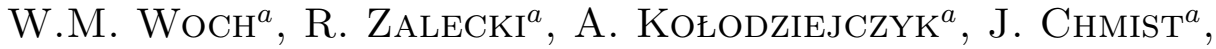 \\ O. HEIML ${ }^{b}$ AND G. GRITZNER ${ }^{b}$ \\ ${ }^{a}$ Faculty of Physics and Applied Computer Science \\ AGH University of Science and Technology \\ al. Mickiewicza 30, 30-059 Kraków, Poland \\ ${ }^{b}$ Institut für Chemische Technologie Anorganischer Stoffe \\ Johannes Kepler Universität, 4040 Linz, Austria
}

\begin{abstract}
The temperature and magnetic field dependences of a.c. resistance of $c$-axis oriented $\left(\mathrm{Tl}_{0.5} \mathrm{~Pb}_{0.5}\right)\left(\mathrm{Sr}_{0.8} \mathrm{Ba}_{0.2}\right)_{2} \mathrm{Ca}_{2} \mathrm{Cu}_{3} \mathrm{O}_{x}$ and $\left(\mathrm{Tl}_{0.6} \mathrm{~Pb}_{0.24} \mathrm{Bi}_{0.16}\right)\left(\mathrm{Sr}_{0.9} \mathrm{Ba}_{0.1}\right)_{2} \mathrm{Ca}_{2} \mathrm{Cu}_{3} \mathrm{O}_{x}$ thin films as well as of $\left(\mathrm{Tl}_{0.5} \mathrm{~Pb}_{0.5}\right)\left(\mathrm{Sr}_{0.8} \mathrm{Ba}_{0.2}\right)_{2} \mathrm{Ca}_{2} \mathrm{Cu}_{3} \mathrm{O}_{x}$ bulk sample from $77 \mathrm{~K}$ to room temperature and in magnetic fields from zero to 3000 Oe were measured and analyzed. The magnetic field and temperature dependence of the resistive superconducting transition and irreversibility field were discussed both in the flux-creep model and in the superconducting liquid vortex state model. The temperature width of resistive transition was explained taking advantage of the Ambegaokar and Halperin model of the resistance of superconducting Josephson weak links and barrier of vortex motion presented by Tinkham. The irreversibility field was described by an exponential formula.
\end{abstract}

PACS numbers: 74.78.Bz, 74.25.Qt, 74.62.Yb

\section{Introduction}

The problem of understanding of the vortex motion in high temperature superconductors (HTS) is important and debatable since discovery of the materials. The first approaches to the problem were in some sense alternative but the final relation for the temperature dependence of the irreversibility field had the same form 


$$
H_{\text {irr }}=H_{0}\left(1-\frac{T}{T_{\mathrm{c}}}\right)^{3 / 2}
$$

where $H_{0}$ is a constant and $T_{\mathrm{c}}$ is the critical temperature. The first paper [1] employed the model of superconducting grains small enough in comparison to the London penetration depth, which were assumed to form weakly coupled closed loops. This state was called a superconductive glassy state or vortex state. Some essential features are the difference in field-cooled and zero-field-cooled responses, the existence of de Almeida-Thouless [2] line separating metastable from stable regions and the non-exponential time dependence of magnetization. The line determined from the formula (1) was called "irreversibility line" or "quasi de AlmeidaThouless line".

Yeshurun and Malozemoff [3] studied the strong, anisotropic magnetic relaxation and they have interpreted it with the thermally activated flux-creep model. They found out that the flux activation energy $U$ is usually in the order of several electronvolts for conventional second type superconductors while they have obtained $U=0.15 \mathrm{eV}$ for HTS. This low $U$-value and the order of magnitude higher $T_{\mathrm{c}}$ in HTS as compared to conventional superconductors leads to the observed "giant" flux creep. They obtained also the irreversibility line described by the formula (1).

In the Tinkham paper [4] the model proposed by Yeshurun and Malozemoff was extended to account for the experimentally measured width and shape of the resistive transition in magnetic field. Tinkham used the formula

$$
\frac{R}{R_{\mathrm{n}}}=\left[I_{0}\left(\frac{\gamma}{2}\right)\right]^{-2}
$$

where $I_{0}$ is the modified Bessel function usually given by series, $R$ is the resistance of the weak links, $R_{\mathrm{n}}$ is the resistance in normal state at given temperature, $\gamma$ is the parameter concerned with the activation energy of vortex motion. The formula was obtained by Ambegaokar and Halperin [5] for superconducting Josephson weak links. The parameter $\gamma$ obtained has the form

$$
\gamma=\frac{U}{k_{\mathrm{B}} T}=\frac{A(1-t)^{3 / 2}}{H},
$$

where the coefficient $A$ depends on a critical current at zero magnetic field and $t=T / T_{\mathrm{c}} . H$ is the dc magnetic field. If we put the formula (3) into Eq. (2) and if we take only two first terms of the Bessel series we can rewrite

$$
\frac{R}{R_{\mathrm{n}}}=\left[I_{0}\left(\frac{A(1-t)^{3 / 2}}{2 H}\right)\right]^{-2}=\left[1-\left(\frac{A(1-t)^{3 / 2}}{4 H}\right)^{2}\right]^{-2},
$$

for the dc magnetic field $H \neq 0$. Equation (2) is valid for each value of $R / R_{\mathrm{n}}$. If we consider that $R / R_{\mathrm{n}}=0$, then a simple calculation gives us the following results 
for the width of resistive transition:

$$
\Delta T=16 T_{\mathrm{c}}\left(\frac{1}{A}\right)^{2 / 3} H^{2 / 3}=C H^{2 / 3} .
$$

For HTS, even for $H=0$ the resistive transition is relatively wide, so $\Delta T \neq 0$. Therefore, Eq. (5) should be rewritten to the following form:

$$
\Delta T=C H^{n}+\Delta T_{0},
$$

where $n=2 / 3$ and $\Delta T_{0}$ means the width of the resistive transition at zero d.c. applied magnetic field. The relation (6) will be used to fit the experimental data.

\section{Experimental}

The preparation procedure of our thin films: $\left(\mathrm{Tl}_{0.5} \mathrm{~Pb}_{0.5}\right)\left(\mathrm{Sr}_{0.8} \mathrm{Ba}_{0.2}\right)_{2} \mathrm{Ca}_{2} \mathrm{Cu}_{3} \mathrm{O}_{x}$ (sample no. 1) $\left(\mathrm{Tl}_{0.6} \mathrm{~Pb}_{0.24} \mathrm{Bi}_{0.16}\right)\left(\mathrm{Sr}_{0.9} \mathrm{Ba}_{0.1}\right)_{2} \mathrm{Ca}_{2} \mathrm{Cu}_{3} \mathrm{O}_{x}$ (sample no. 2 ) $\left(\mathrm{Tl}_{0.5} \mathrm{~Pb}_{0.5}\right)\left(\mathrm{Sr}_{0.8} \mathrm{Ba}_{0.2}\right)_{2} \mathrm{Ca}_{2} \mathrm{Cu}_{3} \mathrm{O}_{x}$ (bulk sample) were described in the papers [6-8].

The prepared samples were characterized by several methods. X-ray diffraction employing Ni-filtered $\mathrm{Cu} K_{\alpha}$ radiation has been carried out on a Geigerflex IIa (Rigaku, Japan) and an X'Pert (Pananalytical). The texture analysis was carried out emploing an X'Pert (Pananalytical). Optical microscopy in polarized light in combination with a Laves Ernst compensator was carried out on an Axioplan (Zeiss, Germany). All these methods showed good quality of the specimens with the single phase stoichiometric compositions.

The measurements of resistance vs. temperature and magnetic field were carried out using the four probes a.c. method. The electrical contacts were made by silver paint. The resistance of the contacts were in the order of $5 \Omega$. The sample was placed in the centre of copper coils, which produced the d.c. magnetic field controlled by the Hall probe with 2 Gs accuracy. The thallium bulk sample is the plate with $9 \times 1.5 \times 1 \mathrm{~mm}$ dimensions. The thin film $\left(\mathrm{Tl}_{0.5} \mathrm{~Pb}_{0.5}\right)\left(\mathrm{Sr}_{0.8} \mathrm{Ba}_{0.2}\right)_{2} \mathrm{Ca}_{2} \mathrm{Cu}_{3} \mathrm{O}_{x}$ sample dimensions are $10 \mathrm{~mm} \times 4 \mathrm{~mm} \times 0.85 \mu \mathrm{m}$. The $c$-axis was parallel to the d.c. magnetic field. The thin film $\left(\mathrm{Tl}_{0.6} \mathrm{~Pb}_{0.24} \mathrm{Bi}_{0.16}\right)\left(\mathrm{Sr}_{0.9} \mathrm{Ba}_{0.1}\right)_{2} \mathrm{Ca}_{2} \mathrm{Cu}_{3} \mathrm{O}_{x}$ dimensions are $0.4 \mathrm{~mm} \times 9 \mathrm{~mm} \times 0.8 \mu \mathrm{m}$. The temperature was monitored by a Lake Shore temperature controller with a chromel-gold $0.07 \%$ iron thermocouple from $77 \mathrm{~K}$ to $300 \mathrm{~K}$ with an accuracy of $0.1 \mathrm{~K}$.

\section{Results and discussion}

The measurements of the resistance vs. temperature for different values of d.c. applied magnetic field are shown in Fig. 1. From these measurements two important values are obtained: the width of resistive transition and the irreversibility field. The width of the resistive transitions $\Delta T$ (between $10 \%$ and $90 \%$ of normal state resistance just above transition temperature $T_{\mathrm{c}}$ ) versus d.c. applied magnetic field are shown in Fig. 2. The width increases as a function of applied magnetic 

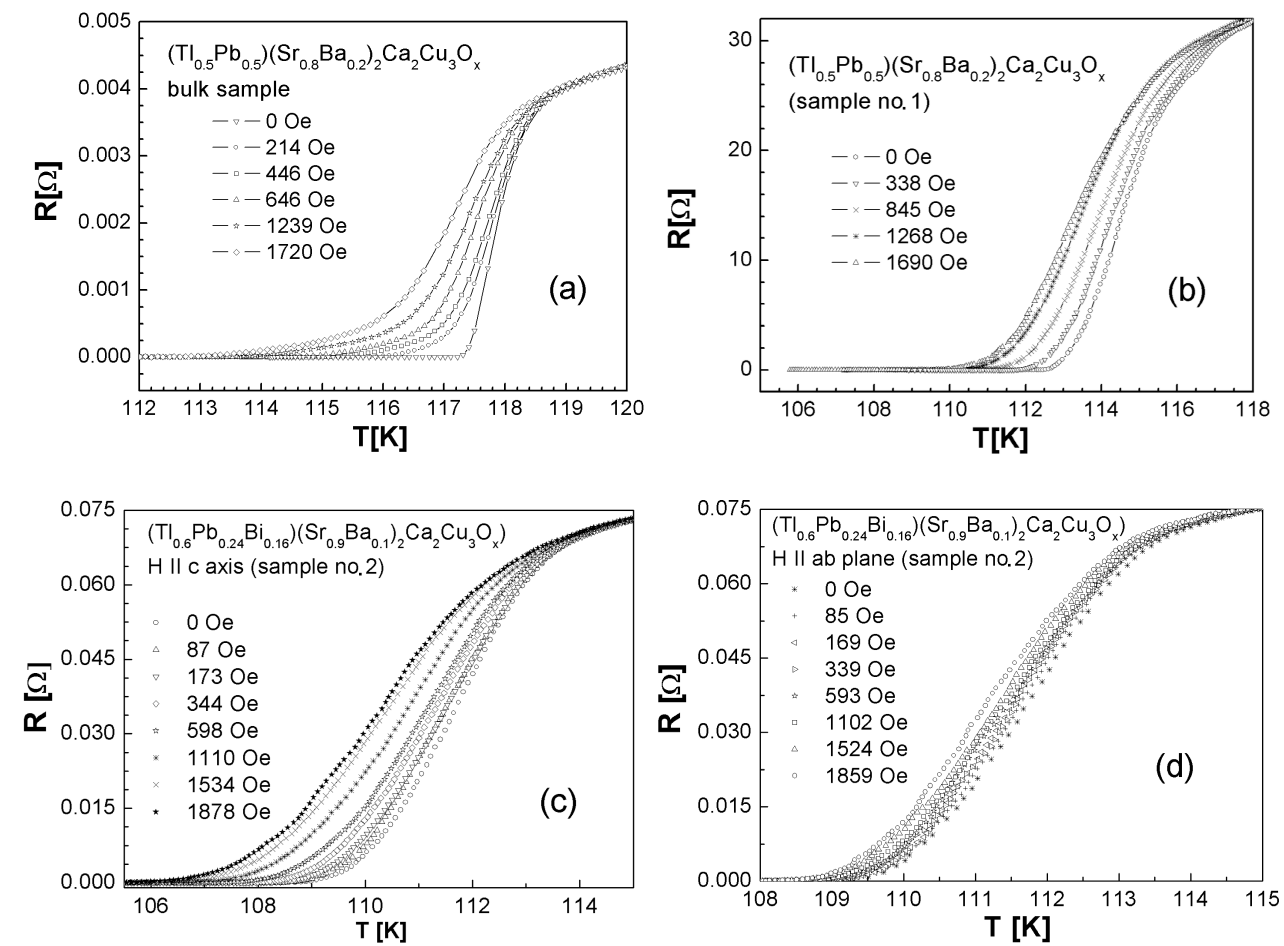

Fig. 1. The resistance vs. temperature for different values of d.c. applied magnetic field for: (a) thallium bulk sample, (b) thallium thin sample no. 1, (c) thallium thin film sample no. 2 for d.c. applied field $H \| c$ axis, (d) thallium thin film sample no. 2 for d.c. applied field $H \| a b$ plane.

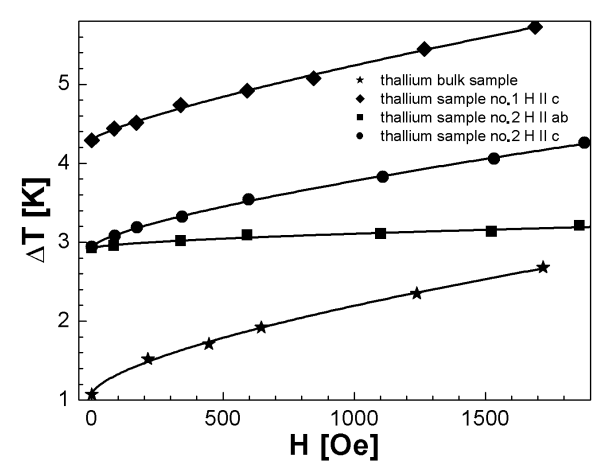

Fig. 2. The temperature width of the resistive transitions $\Delta T$ versus d.c. applied magnetic field.

field and its value changes from about $1 \mathrm{~K}$ for thallium bulk sample to $6 \mathrm{~K}$ for thallium sample no. 1 (magnetic field $H \| c$ axes). The resistive transition $\Delta T$ as 
a function of magnetic field was fitted using formula (6) which has two parameters: $C$ and $\Delta T_{0}$. In this paper we have assumed that also exponent in formula (6) may be changed so the formula (6) with three parameters was fitted to the experimental data. The exponent in Eq. (6) is close to $2 / 3$ for bulk thallium sample. The exponents of thallium thin films are for sample no. $1, n=0.79$ and for sample no. 2 , $n=0.71$ for perpendicular orientation and $n=0.59$ for parallel orientation. The fitted widths are very close to experimental ones and are listed in Table. These

TABLE

The critical temperature $T_{\mathrm{c}}$, the experimental and calculated temperature width of transition $\Delta T_{0}$ and the calculated parameters $C, H_{0}$, and $n$ from Eqs. (6) and (7).

\begin{tabular}{|c|c|c|c|c|c|c|}
\hline \multirow{2}{*}{$\begin{array}{l}T_{\mathrm{c}} \\
{[\mathrm{K}]}\end{array}$} & \multicolumn{2}{|c|}{$\Delta T_{0}[\mathrm{~K}]$} & \multirow{2}{*}{$\begin{array}{l}C \text { from } \\
\text { fitting }\end{array}$} & \multirow[t]{2}{*}{$H_{0}[\mathrm{kOe}]$} & \multicolumn{2}{|c|}{$n$ from } \\
\hline & exp. & fit. & & & Eq. (6) & Eq. (7) \\
\hline \multicolumn{7}{|c|}{$\left(\mathrm{Tl}_{0.5} \mathrm{~Pb}_{0.5}\right)\left(\mathrm{Sr}_{0.8} \mathrm{Ba}_{0.2}\right)_{2} \mathrm{Ca}_{2} \mathrm{Cu}_{3} \mathrm{U}_{0.01} \mathrm{O}_{x}$ bulk sample } \\
\hline 117.3 & $1.1 \pm 0.1$ & $1.08 \pm 0.03$ & $0.012 \pm 0.002$ & $280 \pm 20$ & $0.65 \pm 0.03$ & $1.63 \pm 0.03$ \\
\hline \multicolumn{7}{|c|}{ thin film (sample no. 1) $\left(\mathrm{Tl}_{0.5} \mathrm{~Pb}_{0.5}\right)\left(\mathrm{Sr}_{0.8} \mathrm{Ba}_{0.2}\right)_{2} \mathrm{Ca}_{2} \mathrm{Cu}_{3} \mathrm{O}_{x} H \| c$} \\
\hline 114.3 & $4.3 \pm 0.1$ & $4.30 \pm 0.03$ & $0.010 \pm 0.004$ & $450 \pm 140$ & $0.79 \pm 0.04$ & $1.61 \pm 0.09$ \\
\hline \multicolumn{7}{|c|}{ thin film (sample no. 2) $\left(\mathrm{Tl}_{0.6} \mathrm{~Pb}_{0.24} \mathrm{Bi}_{0.16}\right)\left(\mathrm{Sr}_{0.9} \mathrm{Ba}_{0.1}\right)_{2} \mathrm{Ca}_{2} \mathrm{Cu}_{3} \mathrm{O}_{x} H \| a b$} \\
\hline 111.6 & $2.94 \pm 0.1$ & $2.92 \pm 0.03$ & $0.008 \pm 0.007$ & $7000 \pm 2500$ & $0.59 \pm 0.14$ & $1.68 \pm 0.07$ \\
\hline \multicolumn{7}{|c|}{ hin film (sample no. 2) $\left(\mathrm{Tl}_{0.6} \mathrm{~Pb}_{0.24} \mathrm{Bi}_{0.16}\right)\left(\mathrm{Sr}_{0.9} \mathrm{Ba}_{0.1}\right)_{2} \mathrm{Ca}_{2} \mathrm{Cu}_{3} \mathrm{O}_{x} H \| c$} \\
\hline & & $2.94 \pm 0.01$ & $0.006 \pm 0.001$ & $170 \pm 30$ & $0.71 \pm 0.02$ & $1.33 \pm 0.05$ \\
\hline
\end{tabular}

results show that the approach through the model of resistance of the Josephson weak links [5] could be used to describe the properties of the intergranular weak links of thallium bulk samples which have plate-like grains of $15-20 \mu \mathrm{m}$ long [9]. On the contrary, intergranular properties of the thin film samples are different because they obey relation (6) with different values of exponents than $2 / 3$. At zero applied magnetic field the temperature width of transition is connected with superconducting strength distribution of the weak links. The sample undergoes the transition to superconducting state step by step and at a given temperature only some part of the sample is superconducting. If we turn on the d.c. magnetic field, smaller part of the sample is superconducting at the same temperature. This is the reason of the widening of the temperature width versus d.c. magnetic field.

Equation (1) with exponents $3 / 2$ is connected with the vortex motion [2-5]. The relation is quite different for the temperature dependence of upper critical field which obeys linear [10] or parabolic [11] temperature dependence. The temperature dependence of reversibility field shows upward (negative) curvature and the reversibility line is usually described by the following equation:

$$
H_{\mathrm{irr}}=H_{0}\left(1-\frac{T}{T_{\mathrm{c}}}\right)^{n}
$$




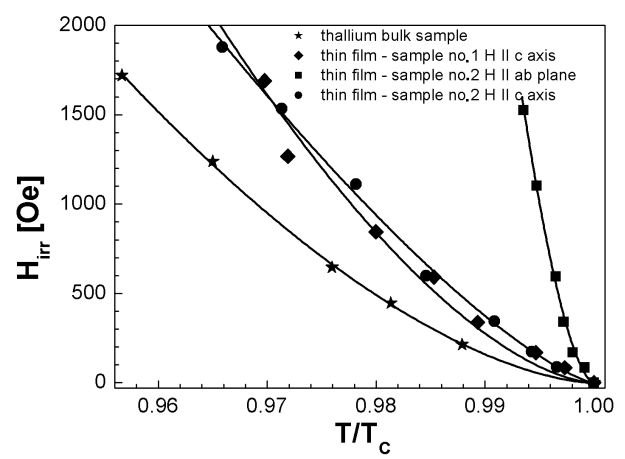

Fig. 3. The irreversibility field as a function of reduced temperature.

where $H_{0}$ is the reversibility field at $0 \mathrm{~K}$ and exponent $n$ can be 1.5 or higher. The Houghton et al. approach [12] based on thermodynamic melting of vortex lattice predicts $n=2$ and some experimental data [13] is in a good agreement with that prediction. In this paper we have assumed that the temperature at which the whole sample stayed superconducting (resistance is equal to zero) separates the reversibility from irreversibility region i.e. the vortex glass from liquid vortex state. These temperatures were extracted from our magnetoresistance measurements and they were fitted by Eq. (7) using two parameters: $H_{0}$ and $n$ (see Fig. 3). The parameter $n$ obtained from the fitting procedure is generally greater than $3 / 2$. Only for the thin film sample no. 2 in perpendicular orientation $n=1.33 \pm 0.05$ and it is significantly lower than $3 / 2$. The exponents for all samples are collected in Table. One may conclude that the upward curvature for these samples is generally stronger than for line described by Eq. (1).

\section{Conclusions}

The conclusions of the paper are twofold:

1. The d.c. applied magnetic field widens the resistive transition and these dependencies were successfully fitted by the formula: $\Delta T=C H^{n}+\Delta T_{0}$. The parameter $n$ is generally bigger than $2 / 3$. The fitted parameters $\Delta T_{0}$ perfectly correspond to experimental ones.

2. The temperatures at which the whole sample stayed superconducting separate the vortex glass from liquid vortex state and they were well described by the irreversibility field vs. temperature with different exponent $n$ (see Eq. (7)). 


\section{Acknowledgments}

This work was financially supported by the Faculty of Physics and Applied Computer Science, AGH University of Science and Technology, Cracow, through the statutory funds from the State Committee for Scientific Research (Poland). The support by the Austrian Exchange Service (ÖAD) Scientific \& Technological Cooperation Austria-Poland (project number 7/2003) is also appreciated.

\section{References}

[1] K.A. Müller, M. Takashige, J. Bednorz, Phys. Rev. Lett. 58, 1143 (1987).

[2] J.R.L. de Almeida, D.J. Thouless, J. Phys. A 11, 983 (1978).

[3] Y. Yeshurun, A.P. Malozemoff, Phys. Rev. Lett. 60, 2202 (1988).

[4] M. Tinkham, Phys. Rev. Lett. 61, 1658 (1988).

[5] V. Ambegaokar, B.I. Halperin, Phys. Rev. Lett. 22, 1364 (1969).

[6] E. Kuzmann, M. Mair, Z. Klencsár, A. Vértes, G. Gritzner, J. Radioanal. Nucl. Chem. 246, 107 (2000).

[7] M. Eder, G. Gritzner, Supercond. Sci. Technol. 13, 1302 (2000).

[8] O. Heiml, G. Gritzner, Supercond. Sci. Technol. 15, 956 (2002).

[9] M. Mair, W.T. König, G. Gritzner, Supercond. Sci. Technol. 8, 894 (1995); W.T. König, G. Gritzner, M. Reissner, W. Steiner, Physica C 258, 175 (1996); M. Reissner, W.T. König, W. Steiner, G. Gritzner, Czech. J. Phys. 46, 1757 (1996).

[10] M.W. McElfresh, Y. Yeshurun, A.P. Malozemoff, F. Holtzberg, Physica A 168, 308 (1990); and references therein.

[11] W.M. Woch, T. Ściȩżor, A. Kołodziejczyk, J. Chmist, Z. Trybuła, J. Stankowski, T. Brylewski, K. Przybylski, J. Magn. Magn. Mater. 140-144, 1311 (1995).

[12] A. Houghton, P.A. Pelcovits, A. Sudbo, Phys. Rev. B 40, 6763 (1989).

[13] J. Ricketts, R. Puźniak, C.-J. Liu, G.D. Gu, N. Koshizuka, H. Yamauchi, Appl. Phys. Lett. 65, 3284 (1999). 